\title{
Low Complexity Receiver Design for MIMO-Radar
}

\author{
Sajid Ahmed and Mohamed-Slim Alouini \\ Electrical Engineering Program \\ King Abdullah University of Science and Technology (KAUST) \\ Thuwal, Makkah Province, Saudi Arabia. \\ Emails: \{sajid.ahmed,slim.alouini\}@kaust.edu.sa.
}

\begin{abstract}
In this work, an algorithm for the multiple-input multiple-output (MIMO) radar is proposed. It has low computational complexity compared to the available schemes, and relatively low side-lobe-levels in the receive beampattern compared to the phased-array and MIMO-radar. In the proposed algorithm, the received signal vector of MIMO-radar is divided into subvectors, and each sub-vector is multiplied with the corresponding weight vector. The number of sub-vectors and weight vectors are optimally found to maximise the received signal power from the target of interest direction. The proposed scheme can be effectively applied in passive radars to minimise the side-lobe levels and place deep nulls for interferers in the receive beampattern. Simulation results show that the proposed scheme has relatively lower side lobe levels and better detection capabilities compared to MIMOradar and phased-array.
\end{abstract}

Index Terms-Colocated antennas, MIMO-radar partitioning.

\section{INTRODUCTION}

Recently several researchers have considered the application of multiple-input multiple-output (MIMO) techniques developed for wireless communication systems to the radar systems. In MIMO communication systems, $n_{T}$ antennas are deployed at the transmitter and $n_{R}$ antennas at the receiver to increase the data rate and provide multiple paths to mitigate the fading in the channel [1]-[3]. Like MIMO communications, which revolutionized the design, development and deployment of wireless networks over the last decade, MIMO-radar offers a new paradigm for signal processing research. MIMO-radars have many advantages over their phased-array counterparts: improved spatial resolution; better parametric identifiability, and greater flexibility to achieve the desired beampattern [4].

MIMO-radars can be classified into two categories: widely distributed [1] and colocated [2]. In the widely distributed case the transmitting antennas are separated so that each antenna may view a different aspect of the target. This topology provides spatial diversity. In colocated systems the transmitting antennas are spaced so that all the transmit antennas view the same aspect of the target. The colocated antenna radar cannot provide spatial diversity but can increase the spatial resolution of the system. In contrast to phased-array, MIMO-radar allow each transmitting antenna to transmit independent waveforms, which provide extra degrees-of-freedom (DOF) that can be exploited to improve system performance [5], [6].

In phased-array radars, the transmitted signals are coherent between different elements of the array that yields gain in signal-to-noise ratio (SNR) but it has poor parametric identifiability problem. MIMO-radar has better parametric identi- fiability but compared to phased-array radar it shows loss in SNR due to non-coherent processing. To exploit the benefits of both MIMO-radar and phased-array the available antennas are configured in number of ways. The configuration in [7] uses multiple independent phased-arrays at widely separated locations. The coherent processing between the individual phasedarray at the fusion center is a practical issue. The configuration in [8] divides the given transmit antennas into $K$ overlapping sub-arrays, where $1 \leq K \leq n_{T}$. Each sub-array transmits the waveform, which is orthogonal to the waveforms transmitted by the other sub-arrays. The phased-MIMO configuration has lower angular resolution and requires different powers to be transmitted from different antennas. If each antenna is required to transmit at a different power level then, for maximum power efficiency, multiple different Radio-frequency amplifiers (RFA)'s with different bias voltage levels will be required. A better solution is to have identical RFA's all working at the same maximum power level.

In contrast to phased-MIMO scheme, in our proposed scheme the $n_{T} n_{R} \times 1$ received signal vector is sub-divided into $K$ overlapping sub-vectors, where in contrast to phasedMIMO $1 \leq K \leq n_{T} n_{R}$. Each sub-vector is multiplied with different weight vector and a new steering vector is formed and processed. The weight vectors and the number of sub-vectors are optimally derived to maximise the received-signal-power. Thus, in contrast to phased-MIMO this scheme does not have equal power problem at the transmitter, has potential for better angular resolution and offers a lower complexity.

The remainder of this paper is organised as follows. In the following section the problem formulation and some background are given. The proposed algorithm is developed in section III and the beamformer for the receiver is designed in section IV. Simulation results are given in section V, followed by our conclusions in section VI.

Notation: Bold upper case letters, $\mathbf{X}$, and lower case letters, $\mathbf{x}$, respectively denote matrices and vectors. The $m$ th column vector of a matrix $\mathbf{X}$ is denoted by $\mathbf{x}_{m}$. The identity matrix of dimension $N \times N$ is denoted by $\mathbf{I}_{N}$. Conjugate transposition of a matrix is denoted by $(.)^{H}$ and statistical expectation is denoted by $\mathrm{E}\{$.$\} .$

\section{Problem Formulation and Previous Work}

Consider a uniform linear array of $n_{T}$ transmit and $n_{R}$ receive antennas. There is a target of interest, which is located at an angle $\theta_{t}$ and there are $L$ interferers located at angles $\theta_{1}$ to 
$\theta_{L}$. For better detection, the receiver should be able to maximise the received power from the target direction and minimise from all the other directions. In addition to this, if interferers are present then the receiver should be able to place deep nulls in the direction of interferers. To design such receiver, consider $x_{m}(n)$ is the baseband signal transmitted from antenna $m$ and $d$ is the inter-element-spacing (IES), in terms of number of halfwavelengths, between any two adjacent antennas. For example, if $d=1$ then IES between two adjacent antennas will be $\lambda / 2$ and for $d=2$ it will will be $\lambda$, where $\lambda$ is the wavelength of the transmitted waveform. The baseband reflected-signal from the target/interferer at location $\theta_{p}$ can be written as

$r_{p}(n)=\sum_{m=1}^{n_{T}} \beta_{m} e^{j(m-1) \pi d \sin \left(\theta_{p}\right)} x_{m}(n), \quad n=1,2, \ldots, N$

where $\beta_{m}$ is the reflection coefficient of the target/interferer. By defining $\mathbf{a}_{T}\left(\theta_{p}\right)=\left[\begin{array}{lll}1 & e^{j \pi d \sin \left(\theta_{p}\right)} \cdots e^{j\left(n_{T}-1\right) \pi d \sin \left(\theta_{p}\right)}\end{array}\right]^{T}, \mathrm{a}$ transmit steering vector corresponding to the target at location $\theta_{p}$, and $\mathbf{x}(n)=\left[\begin{array}{llll}x_{1}(n) & x_{2}(n) & \cdots & x_{n_{T}}(n)\end{array}\right]^{T}$, a vector of transmitted signals from all the antennas at time index $n$, the reflected signal in (1) in vector form can be written as

$$
r_{p}(n)=\mathbf{a}_{T}^{T}\left(\theta_{p}\right) \mathbf{x}(n) .
$$

In MIMO-radar, generally the transmitted symbols from all antennas are fully uncorrelated i.e., $\mathrm{E}\left\{x_{p}(n) x_{q}(n)\right\}=0$, for $p \neq q$. At each receive antenna the received signal is passed through a matched-filter and the sampled signals are correlated with the $n_{T}$ transmitted symbols. By cascading all the signals collected after correlation at each receive antenna, the received signals in vector form can be written as

$$
\mathbf{y}=\beta_{t} \mathbf{a}_{T}\left(\theta_{t}\right) \otimes \mathbf{a}_{R}\left(\theta_{t}\right)+\sum_{i=1}^{L} \beta_{i} \mathbf{a}_{T}\left(\theta_{i}\right) \otimes \mathbf{a}_{R}\left(\theta_{i}\right)+\mathbf{v},
$$

where $\mathbf{a}_{R}\left(\theta_{q}\right)=\left[\begin{array}{llll}1 & e^{j \pi \sin \left(\theta_{q}\right)} & \cdots & e^{j\left(n_{R}-1\right) \pi \sin \left(\theta_{q}\right)}\end{array}\right]^{T}$ is the receive-steering-vector for the target at location $\theta_{q}$ and $\mathbf{v}=\left[\begin{array}{llll}v(0) & v(1) & \cdots & v\left(n_{R} n_{T}-1\right)\end{array}\right]^{T}$ is the vector of circularly symmetric white Gaussian noise samples each of zero mean and $\sigma_{n}^{2}$ variance.

To maximise the signal-to-interference-plus-noise ratio (SINR), in MIMO-radar a beamformer weight vector, b, is designed at the receiver. By multiplying the received signal in (3) with $\mathbf{b}$, we can write

$$
\mathbf{b}^{H} \mathbf{y}=\beta_{t} \mathbf{b}^{H} \mathbf{s}\left(\theta_{t}\right)+\sum_{i=1}^{L} \beta_{i} \mathbf{b}^{H} \mathbf{s}\left(\theta_{i}\right)+\mathbf{b}^{H} \mathbf{v}
$$

where $\mathbf{s}\left(\theta_{q}\right)=\mathbf{a}_{T}\left(\theta_{q}\right) \otimes \mathbf{a}_{R}\left(\theta_{q}\right)$ is a virtual steering vector corresponding to the target/interferer at location $\theta_{q}$. From (4) the SINR can be defined as

$$
\operatorname{SINR}=\frac{\left|\beta_{t} \mathbf{b}^{H} \mathbf{s}\left(\theta_{t}\right)\right|^{2}}{\mathbf{b}^{H} \mathbf{R}_{i n} \mathbf{b}}
$$

where $\mathbf{R}_{\text {in }} \in \mathcal{C}^{n_{T} n_{R} \times n_{T} n_{R}}$ is the covariance matrix of interference plus noise samples and is defined as

$$
\mathbf{R}_{i n}=\underbrace{\sum_{i=1}^{L}\left|\beta_{i}\right|^{2} \mathbf{s}\left(\theta_{i}\right) \mathbf{s}^{H}\left(\theta_{i}\right)}_{\text {Interferer covariance matrix }}+\underbrace{\sigma_{n}^{2} \mathbf{I}_{n_{R} n_{T}}}_{\text {Noise covariance matrix }} .
$$

If $\lambda_{1}, \lambda_{2}, \ldots, \lambda_{n_{R} n_{T}}$ are the eigenvalues, $\boldsymbol{\Lambda}$ is the diagonal matrix of the eigenvalues, and $\mathbf{U}$ is the matrix of eigenvectors of the interferers covariance matrix then the covariance matrix $\mathbf{R}_{\text {in }}$ can be written as

$$
\mathbf{R}_{i n}=\sum_{q=1}^{n_{T} n_{R}}\left(\sigma_{n}^{2}+\lambda_{q}\right) \mathbf{u}_{q} \mathbf{u}_{q}^{H} .
$$

To maximise SINR with respect to b using Schwartz's inequality, (5) can be re-written as

$$
\begin{aligned}
\operatorname{SINR} & =\frac{\left|\beta_{t} \mathbf{b}^{H} \mathbf{R}_{i n}^{1 / 2} \mathbf{R}_{i n}^{-1 / 2} \mathbf{s}\left(\theta_{t}\right)\right|^{2}}{\mathbf{b}^{H} \mathbf{R}_{i n} \mathbf{b}} \\
& \leq \beta_{t}^{2} \frac{\mathbf{b}^{H} \mathbf{R}_{i n} \mathbf{b} \mathbf{s}^{H}\left(\theta_{t}\right) \mathbf{R}_{i n}^{-1} \mathbf{s}\left(\theta_{t}\right)}{\mathbf{b}^{H} \mathbf{R}_{i n} \mathbf{b}} \\
& \leq \beta_{t}^{2} \mathbf{s}^{H}\left(\theta_{t}\right) \mathbf{R}_{i n}^{-1} \mathbf{s}\left(\theta_{t}\right) .
\end{aligned}
$$

Therefore, the optimal value of SINR for $\beta_{t}=1$ will be

$$
\begin{aligned}
\operatorname{SINR}_{\circ} & =\mathbf{s}^{H}\left(\theta_{t}\right) \mathbf{R}_{i n}^{-1} \mathbf{s}\left(\theta_{t}\right), \\
& =\mathbf{s}^{H}\left(\theta_{t}\right) \sum_{q=1}^{n_{T} n_{R}} \frac{\mathbf{u}_{q} \mathbf{u}_{q}^{H}}{\sigma_{n}^{2}+\lambda_{q}} \mathbf{s}\left(\theta_{t}\right) .
\end{aligned}
$$

It can be easily proved that the beamformer weight vector that brings the optimal value of SINR can be written as

$$
\mathbf{b}=\frac{\mathbf{R}_{i n}^{-1} \mathbf{s}\left(\theta_{t}\right)}{\mathbf{s}^{H}\left(\theta_{t}\right) \mathbf{R}_{i n}^{-1} \mathbf{s}\left(\theta_{t}\right)} .
$$

Finding the beamformer weight vector, $\mathbf{b}$, requires the inversion of covariance matrix $\mathbf{R}_{i n}$, which can be computed in $\mathcal{O}\left(n_{R}^{3} n_{T}^{3}\right)$ computations. In the presence of only noise the matrix of eigenvectors, $\mathbf{U}$, can be replaced by an identity matrix, $\mathbf{I}_{n_{T} n_{R}}$, and the maximum value of SINR becomes

$$
\begin{aligned}
\operatorname{SINR}_{\circ} & =\mathbf{s}^{H}\left(\theta_{t}\right)\left(\sum_{q=1}^{n_{T} n_{R}} \frac{\mathbf{i}_{q} \mathbf{i}_{q}^{H}}{\sigma_{n}^{2}}\right) \mathbf{s}\left(\theta_{t}\right), \\
& =\frac{n_{R} n_{T}}{\sigma_{n}^{2}} .
\end{aligned}
$$

In MIMO-radar, if the transmitted waveform from antenna $m$ is $x_{m}(n)=x_{1}(n) e^{-j \pi(m-1) \sin \left(\theta_{t}\right)}$ for $m=1, \ldots, n_{T}$, then all the transmitted waveforms will be fully correlated. Such configuration of MIMO-radar is called a phased-array radar. Here, the matched-filter output samples are correlated with only the transmitted symbol sequence $\left\{x_{1}(n)\right\}$. The collected samples from $n_{R}$ antennas after correlation in vector form can be written as

$$
\mathbf{y}_{p h}=\beta_{t} n_{T} \mathbf{a}_{R}\left(\theta_{t}\right)+\sum_{i=1}^{L} \beta_{i} \mathbf{a}_{T}^{H}\left(\theta_{t}\right) \mathbf{a}_{T}\left(\theta_{i}\right) \mathbf{a}_{R}\left(\theta_{i}\right)+\hat{\mathbf{v}} .
$$


For the phased-array radar the optimal value of SINR using Schwartz's inequality can be easily derived as

$$
\mathrm{SINR}_{\circ}=\hat{\mathbf{s}}^{H}\left(\theta_{t}\right) \hat{\mathbf{R}}_{i n}^{-1} \hat{\mathbf{s}}\left(\theta_{t}\right),
$$

where $\hat{\mathbf{s}}\left(\theta_{t}\right)=n_{T} \mathbf{a}_{R}\left(\theta_{t}\right)$. The optimal value of SINR for the noise only case becomes

$$
\mathrm{SINR}_{\circ}=\frac{n_{R} n_{T}^{2}}{\sigma_{n}^{2}} .
$$

By comparing (7) with (9), it can be noted that the SINR of phased-array is better than MIMO-radar. To accomplish the benefits of both MIMO and phased-array the concept of phasedMIMO is introduced in [8]. In this work the $n_{T}$ transmit antennas are divided into $K$ uniform sub-arrays, where $1 \leq K \leq n_{T}$. Here, all the antennas in the sub-array $k$ transmit the waveforms coherently and an antenna in the sub-array $k$ can contribute to other sub-arrays. For the noise only case the optimal SINR of the phased-MIMO radar is given by [8]

$$
\mathrm{SINR}_{\circ}=\frac{n_{R} n_{T}\left(n_{T}-K+1\right)}{\sigma_{n}^{2}}
$$

In (10), it can be noted that for the noise only case, if $K>$ 1 then, the SINR of phased-MIMO radar is better than the MIMO-radar but poorer than the phased-array radar. Moreover, the phased-MIMO radar has lower side-lobe levels compared to the MIMO and phased-array radar. The phased-MIMO scheme has few drawbacks as well which are given below

- Each antenna transmits different power, which requires different power amplifiers for different antennas.

- Introduction of phased-array decreases the parametric identifiability [5] of phased-MIMO.

Due to the drawbacks of phased-MIMO, in the following, we propose a scheme which has low-complexity and relatively lower side-lobe levels for passive-radar applications.

\section{PROPOSED MIMO-RADAR FORMULATION}

In the proposed MIMO-radar scheme the $n_{T} n_{R} \times 1$ virtual steering vector of MIMO-radar is sub-divided into $K$ subvectors. The elements of one sub-vector are allowed to contribute to other sub-vectors as well, or the vectors can partially overlap each other. If all the sub-vectors are uniform i.e., each sub-vector contains the same number of elements then the total number of sub-vectors $K$ may lie between 1 and $n_{T} n_{R}$. For maximum partial overlapping, the total number of elements in each sub-vector for given $K$ will be $n_{T} n_{R}-K+1$. By multiplying each of these sub-vectors with the corresponding weight vectors the proposed received signal model can be written as

$$
\overline{\mathbf{y}}=\underbrace{\beta_{t} \overline{\mathbf{s}}\left(\theta_{t}\right)}_{\text {signal-term }}+\underbrace{\sum_{i=1}^{L} \beta_{i} \overline{\mathbf{s}}\left(\theta_{i}\right)+\overline{\mathbf{v}}}_{\text {interference-plus-noise term }},
$$

where $\overline{\mathbf{s}}\left(\theta_{l}\right)=\left[\begin{array}{llll}\overline{\mathbf{w}}_{1}^{H} \overline{\mathbf{s}}_{1}\left(\theta_{l}\right) & \mathbf{w}_{2}^{H} \overline{\mathbf{s}}_{2}\left(\theta_{l}\right) & \ldots & \overline{\mathbf{w}}_{K}^{H} \overline{\mathbf{s}}_{K}\left(\theta_{l}\right)\end{array}\right]^{T}$, $\overline{\mathbf{v}}=\left[\begin{array}{llll}\overline{\mathbf{w}}_{1}^{H} \overline{\mathbf{v}}_{1} & \overline{\mathbf{w}}_{2}^{H} \overline{\mathbf{v}}_{2} & \cdots & \overline{\mathbf{w}}_{K}^{H} \overline{\mathbf{v}}_{K}\end{array}\right]^{T}$, and $\overline{\mathbf{w}}_{k}$ is the weight vector, while sub-vectors $\overline{\mathbf{s}}_{k}\left(\theta_{l}\right)$ and $\overline{\mathbf{v}}_{k}$ respectively contains the $k$ to $\left(n_{T} n_{R}-K+k\right)$ samples of virtual-steering-vector $\mathbf{s}\left(\theta_{l}\right)$ and noise vector $\mathbf{v}$ of MIMO radar signal model given in (4). This sub-vector division and multiplication with the weight vectors, as a consequence, changes the variance of the noise to $\mathrm{E}\left\{\overline{\mathbf{v}} \overline{\mathbf{v}}^{H}\right\}=\overline{\mathbf{W}} \overline{\mathbf{W}}^{H}$, where

$$
\overline{\mathbf{W}}=\left[\begin{array}{cccc}
\overline{\mathbf{w}}_{1}^{H} & 0 & \cdots & 0 \\
0 & \overline{\mathbf{w}}_{2}^{H} & \ddots & \vdots \\
\vdots & \ddots & \ddots & 0 \\
0 & \cdots & 0 & \overline{\mathbf{w}}_{K}^{H}
\end{array}\right] \in \mathcal{C}^{K \times n_{R} n_{T}} .
$$

The weight matrix, $\overline{\mathbf{W}}$, can be chosen to maximise the signalpower subject to the constraint $\overline{\mathbf{W}}^{H} \mathbf{W}=\mathbf{I}_{K \times K}$, to not change the noise variance. The derivation of such $\overline{\mathbf{W}}$ is left for our future work.

To maximise the energy of the signal-term in (11), the use of Schwartz inequality suggests that

$$
\overline{\mathbf{w}}_{k}=\overline{\mathbf{s}}_{k}\left(\theta_{t}\right) \text { for } k \in\{1,2, \ldots, K\} .
$$

If the weight vectors, $\overline{\mathbf{w}}_{k} \mathrm{~s}$, are chosen to maximise the energy in the signal-term then the maximum energy in the signal-term will be $K\left(n_{T} n_{R}-K+1\right)^{2}$. Since the maximum energy in the signal-term depends on the choice of $K$, its optimum value can be found by minimising the following cost function with respect to $K$

$$
J(K)=-K\left(n_{T} n_{R}-K+1\right)^{2} .
$$

For the minimisation of the cost-function, the differentiation of (12) with respect to $K$ and then equating it equal to zero yields

$$
K=\frac{n_{T} n_{R}+1}{3} \text {. }
$$

Fig. 1 shows the normalised received-signal-power with respect to the value of $K$. For this simulation the number of transmit and receive antenna is 10 . It can be seen in the figure that the received-signal-power is maximum when $K=33$.



Fig. 1. Normalized received-signal-power of the proposed MIMO-radar formulation for given $K$.

Therefore, for the proposed model given in (11), similar to previous cases, the optimal SINR can be found as

$$
\operatorname{SINR}_{\circ}=\overline{\mathbf{s}}^{H}\left(\theta_{t}\right) \overline{\mathbf{R}}_{i n}^{-1} \overline{\mathbf{s}}\left(\theta_{t}\right),
$$

where $\overline{\mathbf{R}}_{\text {in }} \in \mathcal{C}^{K \times K}$ is the covariance matrix of interferenceplus-noise samples. 


\section{BEAMFORMER DESIGN}

For the proposed scheme, the minimum-variancedistortionless response (MVDR) beamformer to maximise the received energy from the target direction and place deep-nulls in the direction of interferers can be derived as

$$
\mathbf{w}_{\text {MVDR }}=\frac{\overline{\mathbf{R}}_{i n}^{-1} \overline{\mathbf{s}}\left(\theta_{t}\right)}{\overline{\mathbf{s}}\left(\theta_{t}\right) \overline{\mathbf{R}}_{i n}^{-1} \overline{\mathbf{s}}\left(\theta_{t}\right)} .
$$

The received power from the direction $\theta, P(\theta)$, can be found as

$$
\mathbf{P}(\theta)=\left|\mathbf{w}_{\mathrm{MVDR}}^{H} \overline{\mathbf{s}}(\theta)\right|^{2} .
$$

The MVDR beamformer can also be used adaptively to find the direction of target from the given signal $\overline{\mathbf{y}}$ in (11) and place deep nulls in the direction of known interferers.

\section{Simulation Results}

In this section to validate the performance of the proposed scheme simulation results are provided. In all of the simulations the number of transmit and receive antennas is 10 and the IES between any two adjacent antenna is half of wavelength, unless otherwise stated. For the fair comparison all systems transmit same power in one simulation. In all of the simulations the target of interest is located at $\theta_{t}=10$-degrees and two interferers are respectively located at $\theta_{1}=-30$-degrees and $\theta_{2}=-10$-degrees.

In the first simulation, the output SINR of the proposed scheme is compared with the phased-array, MIMO-radar, and phased-MIMO schemes. Fig. 2 shows the corresponding SINR performance with respect to SNR of each scheme. It can be seen in the figure that the proposed scheme has poorer SINR compared to the phased-array and phased-MIMO, but has same SINR to that of MIMO-radar due to non-coherent processing at the transmitter. Similarly, Fig. 3 shows the SINR performance using 1 transmit and 20 receive antennas, the total number of elements at the transmitter and receiver are kept same as in the last simulation. The interference-to-noise ratio (INR) is again kept fixed at 30-dB. Such scenarios may exist in passive radars, where the receiver does not have any control on the transmitter of opportunity. It can be seen in the figure that phased-array and phased-MIMO have similar performances, as it was expected due to only one antennas at the transmitter. Since, less power is transmitted in this case, the loss in the SINR in phased-array and phased-MIMO is $18-\mathrm{dB}$, while in the MIMO-radar and proposed algorithm it is only $8-\mathrm{dB}$. In the second simulation the MVDR beamformer is derived for phased-array, MIMO-radar, phased-MIMO and also proposed schemes. The corresponding receive beampatterns are shown in Fig. 4 . It can be seen in the figure that the proposed algorithm outperforms phased-array and MIMO-radar in terms of side-lobe levels. Phased-MIMO scheme has lower side lobe-levels but at the cost of higher computational complexity and poorer angular resolution. The proposed scheme has similar interferer rejection capabilities to that of other schemes. Similarly, Fig. 7 shows the receive beampattern for 1 transmit and 20 receive antennas, for this simulation our proposed scheme outperforms all the others schemes in terms lower side-lobe levels. The computational complexities to find the MVDR beamformer for phased-array, MIMO-radar, phased-MIMO and proposed scheme are respectively $\mathcal{O}\left(n_{R}^{3}\right), \mathcal{O}\left(n_{R}^{3} n_{T}^{3}\right), \mathcal{O}\left(K^{3} n_{R}^{3}\right)$ and $\mathcal{O}\left(\frac{\left(n_{R} n_{T}+1\right)^{3}}{9}\right)$. For example, if $n_{T}=n_{R}=10$, for phased-MIMO the value of $K=5$ and for our proposed scheme $K=33$, therefore, the corresponding complexities are respectively $\mathcal{O}\left(50^{3}\right)$ and $\mathcal{O}\left(33^{3}\right)$. In the final simulation an example of adaptive detection is given. In the simulation three targets are placed at locations -30 -degrees, -10 -degrees and +10 -degrees. The reflection coefficients of targets are 1, 2 and 1.5. For simulation, the number of transmit and receive antennas is 10 , while each antenna transmit 100 BPSK symbols. To detect the target a simple least-squares (LS) estimator can be easily derived for MIMO-radar, phased-MIMO and proposed scheme [4], Fig. 6 shows the corresponding detection performances of the MIMOradar, phased-MIMO and proposed schemes at SNR of 20-dB. It can be seen in the figure that the detection performance of phased-MIMO and proposed scheme are almost same while the detection performance of MIMO-radar is poor. Similarly, Fig. 7 shows the corresponding mean-squared-error (MSE) in the estimation. It can be seen in the figure that the phasedMIMO and proposed scheme have similar MSEs, while the MSE of MIMO-radar is poorer. The advantage of our scheme is that it does not have equal transmit power problem at the transmitter and have lower computational complexity.

\section{CONCLUSION}

A new scheme for the MIMO-radar is proposed, in which $n_{T} n_{R} \times 1$ virtual steering vector is divided into $K$ subvectors. Each sub-vector is multiplied with the weight vector to maximise the received-signal-power from the desired direction and a new virtual steering vector is formed. The number of sub-vectors, $K$, is optimally found to increase the receivedsignal-power. Simulation results have shown that the proposed scheme has low-complexity and relatively lower side-lobe levels compared to the previously known schemes for bi-static radar applications.

\section{REFERENCES}

[1] A. M. Haimovich, R. S. Blum, and L. J. Cimini, "MIMO radar with widely separated antennas," IEEE Signal Processing Mag., vol. 25, pp. 116-129, Jan. 2008.

[2] P. Stoica, J. Li, and X. Zhu, "MIMO radar with co-located antenna: Review of some recent work," IEEE Signal Processing Magazine, vol. 24, pp. 106114, Sep. 2007.

[3] D. R. Fuhrmann and J. S. Antonio, "Transmit beamforming for MIMO radar systems using signal cross-correlation," IEEE Trans. Aerosp. Electron. Syst., vol. 44, pp. 171-185, Jan. 2008.

[4] J. Li and P. Stoica, "MIMO Radar Signal Processing," John, Wiley and Sons, Inc., 2008.

[5] P. Stoica, J. Li, and Y. Xie, "On probing signal design for MIMO radar," IEEE Trans. Signal Processing, vol. 55, pp. 4151-4161, Aug. 2007.

[6] S. Ahmed, J. S. Thompson, and B. Mulgrew, "Finite alphabet constantenvelope waveform design for MIMO radar beampattern," IEEE Trans. on Signal Processing, vol. 59, pp. 5326-5337, Nov. 2011.

[7] L. Xu and J. Li, "Iterative generalised-likelihood ratio test for MIMO radar," IEEE Trans. on Signal Processing, vol. 55, pp. 2375-2385, Jun. 2007.

[8] A. Hassanien and S. A. Vorobyov, "Phased-MIMO Radar: A tradeoff between phased-array and MIMO radars," IEEE Trans. on Signal Processing, vol. 58, pp. 3137-1351, Jun. 2010. 


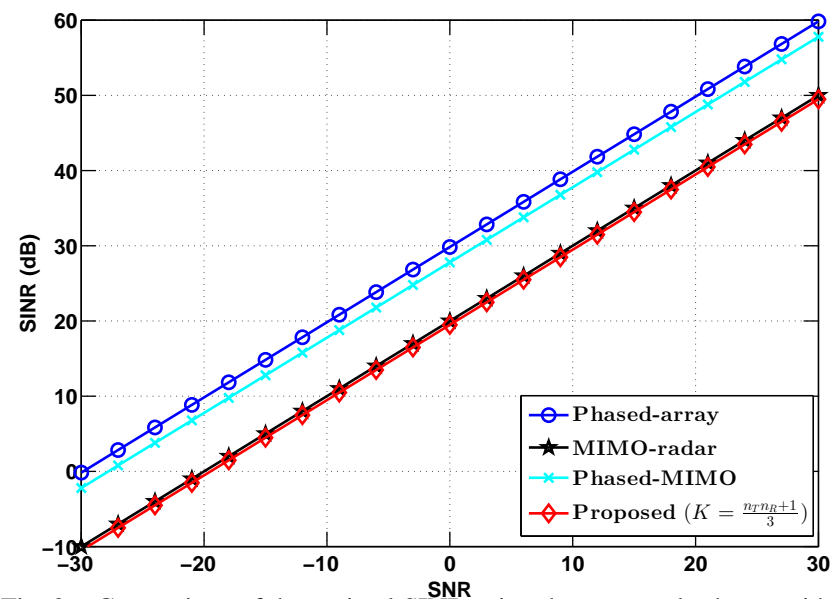

Fig. 2. Comparison of the optimal SINR using the proposed scheme with the phased-array, MIMO-radar, and phased-MIMO schemes. Here, $n_{T}=n_{R}=$ 10 and $\mathrm{INR}=30-\mathrm{dB}$.

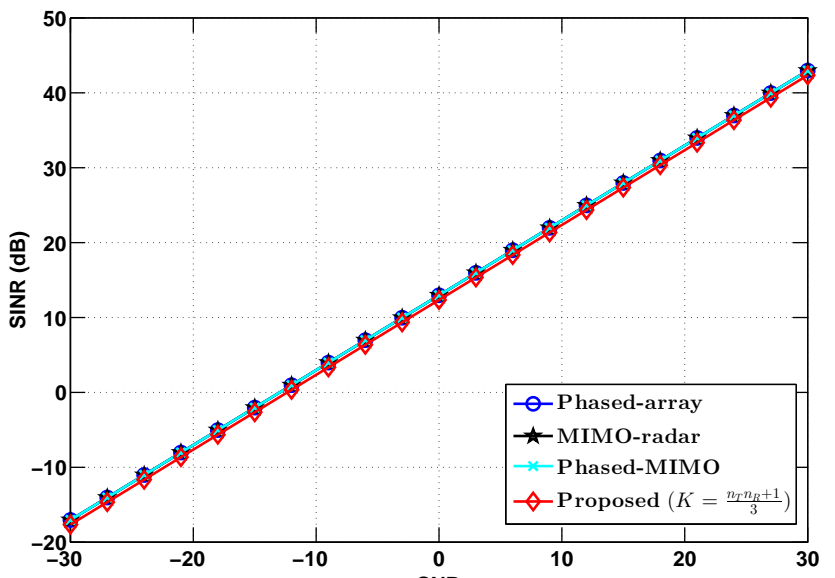

Fig. 3. Comparison of the optimal SINR of the proposed scheme with the MIMO-radar, phased-array and phased-MIMO schemes in the presence of noise and interferences. Here, $n_{T}=1, n_{R}=20$ and INR $=30$-dB.



Fig. 4. Beampattern at the receiver obtained using MVDR beamformer. Here, $n T=n_{R}=10, \mathrm{INR}=50-\mathrm{dB}$ and $\mathrm{SNR}=20-\mathrm{dB}$.

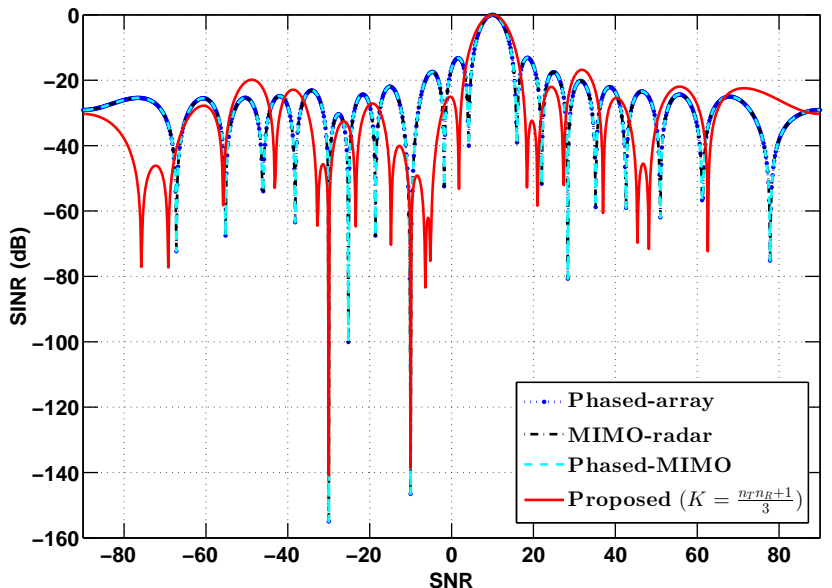

Fig. 5. Beampattern at the receiver obtained using MVDR beamformer. Here, $n_{T}=1, n_{R}=20, \mathrm{INR}=50-\mathrm{dB}$ and SNR $=20-\mathrm{dB}, n_{T}=1$, and $n_{R}=20$.

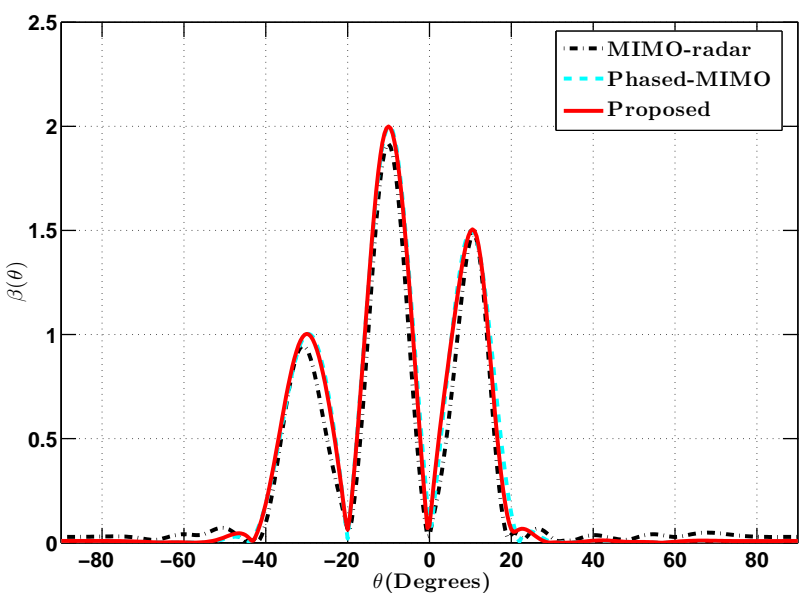

Fig. 6. Estimation performance of MIMO-radar, phased-MIMO and proposed scheme using LS estimator. Here, $\mathrm{SNR}=20 \mathrm{~dB}$.



Fig. 7. MSE between the actual and estimated values of $\beta(\theta)$ obtained using LS estimator for MIMO-radar, phased-MIMO, and proposed schemes. 